Check for updates

Cite this: RSC Adv., 2018, 8, 10865

Received 5th January 2018

Accepted 5th March 2018

DOI: $10.1039 / \mathrm{c} 8 \mathrm{ra00120k}$

rsc.li/rsc-advances

\section{Homogeneous grafting of cellulose with polycaprolactone using quaternary ammonium salt systems and its application for ultraviolet-shielding composite films}

\author{
Yongqi Yu, (D) Xin Gao, Zeming Jiang, (D) Wentao Zhang, Jiwei Ma, Xuejiao Liu \\ and Liping Zhang*
}

Microcrystalline cellulose grafted polycaprolactone (MCC- $g-P C L)$ was successfully synthesized by ringopening copolymerization catalyzed by 4-dimethylaminopyridine in a dual tetrabutylammonium acetate/ dimethyl sulfoxide solvent system. A novel ultraviolet-shielding film based on MCC-g-PCL was prepared by introducing graphene oxide (GO). The results obtained showed that the introduction of GO not only obviously influenced the inherent structure of the MCC-g-PCL but remarkably changed the surface morphology of the composite film. Moreover, the GO/MCC-g-PCL composite showed a significant improvement in tensile strength, from 2.63 to $4.55 \mathrm{MPa}$, as well as elongation-at-break, from $6.4 \%$ to $15.5 \%$, compared with the pure MCC-g-PCL film, owing to the strong hydrogen-bonding interaction that physically crosslinked GO with MCC-g-PCL. Importantly, GO/MCC-g-PCL composite films offered an effective high-energy light-shielding capacity; in particular MCC-g-PCL film containing 1.0 wt\% GO possessed good absorbance between $200 \mathrm{~nm}$ and $300 \mathrm{~nm}$. This study provides a framework for developing cellulose-based ultraviolet-shielding polymers and better understanding the ultravioletshielding mechanism.

\section{Introduction}

Owing to the depletion of the ozone in the Earth's atmosphere, there is a progressive increase in ultraviolet (UV) radiation on human skin. ${ }^{1}$ UV radiation from the Sun or artificial sources causes photodermatosis, acceleration of skin aging and changes to the functioning of the immune system,,$^{2-7}$ and is a prominent risk factor in the development of skin cancer. Thus, prevention measures must be established as UV radiation is increasingly becoming a hazard. The UV-protective properties of cellulose can be improved by UV absorbers, such as zinc oxide (ZnO) nanoparticles, ${ }^{7-10}$ which were incorporated with cellulose, achieving satisfactory UV-protective properties. ${ }^{\mathbf{1 1 - 1 3}}$ The UV protection factor (UPF) values of textiles can be increased by treating them with high concentrations of $\mathrm{ZnO}$ nanoparticles. ${ }^{8}$ However, the excessive use of nanoparticles can lead to ecological problems because the nanoparticles remain in effluent solutions. ${ }^{2}$ On the other hand, $\mathrm{TiO}_{2}$ and $\mathrm{ZnO}$ particles embedded into an organic matrix can photocatalyze the degradation of polymer, ${ }^{\mathbf{1 4}}$ in which case the photostabilization

Department of Material Science and Technology, MOE Key Laboratory of Wooden Material Science and Application, Beijing Forestry University, 100083, Beijing, P. R. China.E-mail: zhanglp418@163.com of the hybrid structure is an additional requirement that must be taken into account.

Recently, Moraes et al. reported a UV-shielding composite film based on graphene oxide (GO) and cellulose acetate (CA) that possessed good absorbance of UV light at wavelengths between $250 \mathrm{~nm}$ and $350 \mathrm{~nm} .{ }^{15}$ However, the mechanical strength was very low possibly due to the weak interaction between oxygen-containing groups on GO and ester groups on CA chains.

Aliphatic polyesters constitute the most versatile group of degradable polymers with good mechanical properties and good biocompatibility, and are particularly interesting as a promising matrix component in bionanocomposites.

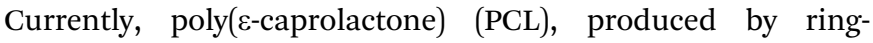
opening polymerization (ROP) of cyclic $\varepsilon$-CL monomer, ${ }^{16-19}$ represents the most typical biodegradable and biobased polymer on the market owing to its cost-competitiveness compared with conventional non-biodegradable polymers. ${ }^{20}$ Cellulose- $g$ PCL has been used in many applications, such as selfassembled nanomicelles in drug delivery and surface-grafted cellulose nanocrystals for reinforcement. ${ }^{21,22}$ However, few monomers were attached to crystalline regions of cellulose in the heterogeneous synthesis process, owing to hydrogen bonds between the hydroxyl groups. ${ }^{23}$ Therefore, it is of great importance to obtain homogeneous cellulose- $g$-PCL with controlled 


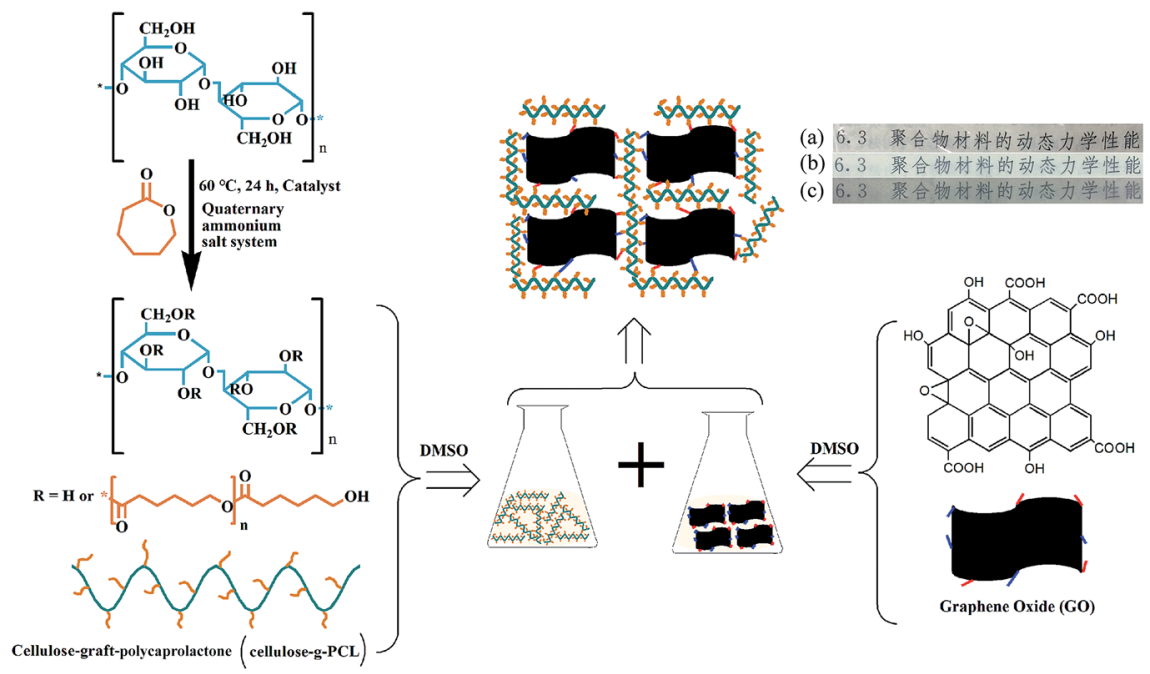

Fig. 1 Scheme outlining the synthesis of MCC-g-PCL and the fabrication of GO/MCC-g-PCL hybrid films by solvent casting: (a) MCC film, (b) MCC-g-PCL film and (c) GO/MCC-g-PCL film.

molecular side-chains. However, it is considerably energyconsuming to do this in 1-butyl-3-methylimidazolium chloride $(\mathrm{BmimCl})$ even at a temperature of $130{ }^{\circ} \mathrm{C}$ for $24 \mathrm{~h} \cdot{ }^{24}$ Moreover, the synthesis conditions in tetrabutylammonium fluoride (TBAF)/dimethyl sulfoxide (DMSO) are vague. ${ }^{25}$

In the present study, MCC- $g$-PCL was homogeneously prepared in tetrabutylammonium acetate (TBAA)/DMSO solvent at $60{ }^{\circ} \mathrm{C}$ (Fig. 1). And the MCC- $g$-PCL film was crosslinked using GO crosslinker under homogeneous conditions using DMSO as solvent. The structure, morphology and mechanical properties of the modified cellulose film were then studied. Finally, the protection of GO/MCC- $g$-PCL against UV light was investigated.

\section{Experimental}

\subsection{Materials}

Avicel powder with a degree of polymerization of 200 was provided by Sinopharm Chemical Reagent Co., Ltd, PR China. The monomer $\varepsilon$-caprolactone with a purity of $99.9 \%$ used in the preparation of cellulose graft copolymers was obtained from Shandong Xiya Chemical Co., Ltd, PR China, and was used as received without any further purification. The tetrabutylammonium acetate (TBAA) $(\geq 90.0 \%)$ and tetrabutylammonium fluoride hydrate $\left(\mathrm{TBAF} \cdot 3 \mathrm{H}_{2} \mathrm{O}\right)(\geq 98.0 \%)$ used as solvent for the cellulose solubilization were purchased from Tokyo Chemical Industry Co., Ltd, Japan. The dimethyl sulfoxide used as assistant solvent for the dissolution of cellulose was of analytical reagent grade and obtained from Beijing Chemical Works, PR China. 4-Dimethylaminopyridine (DMAP) and tin(II) ethylhexanoate of $\geq 95 \%$ and $\geq 99 \%$ purity, respectively, were supplied by Shanghai Macklin Biochemical Co., Ltd, PR China.

\subsection{Preparation of microcrystalline cellulose-grafted polycaprolactone (MCC-g-PCL) and its composite materials}

TBAA and DMSO were mixed in a mass ratio of $2: 8$ at $60{ }^{\circ} \mathrm{C}$. Cellulose pulp was added slowly into the solvent medium. The mixture of cellulose/TBAA/DMSO was stirred at $60{ }^{\circ} \mathrm{C}$ for up to 30 min until a colorless solution was obtained. A solution of $13 \mathrm{~mL} \varepsilon$-caprolactone containing $1.35 \mathrm{~g}$ DMAP was added to $20 \mathrm{~g}$ of $6 \mathrm{wt} \%$ cellulose solution. Polymerization was carried out for $24 \mathrm{~h}$ at $60{ }^{\circ} \mathrm{C}$. After isolation, the polymer was washed and freeze-dried for $48 \mathrm{~h}$.

After $105 \mathrm{mg}$ GO was dispersed in 11.90 DMSO by ultrasonication for $12 \mathrm{~h}$ at room temperature, $2.10 \mathrm{~g}$ of the MCC- $g$ PCL prepared above was added and dissolved in the dispersion. Then GO/MCC- $g$-PCL composite film was prepared via the solvent immersion casting process and finally obtained after drying for $24 \mathrm{~h}$ in an air drying oven at $25^{\circ} \mathrm{C}$.

\subsection{Structure characterization of MCC- $g$-PCL}

The contents of grafted PCL in MCC-g-PCL copolymers were characterized by ${ }^{1} \mathrm{H}$ nuclear magnetic resonance (NMR) spectroscopy. ${ }^{1} \mathrm{H}$ NMR spectra were recorded at $400 \mathrm{MHz}$ using a Bruker BioSpin NMR spectrometer (Bruker Company, Germany) in dimethyl sulfoxide- $\mathrm{d}_{6}\left(\right.$ DMSO- $\left._{6}\right)$ solvent. The chemical shifts were calibrated against the internal standard signals of tetramethysilane (TMS). ${ }^{13} \mathrm{C}$ NMR spectra were acquired on a Bruker BioSpin NMR apparatus at $100 \mathrm{MHz}$, at ambient temperature, using TMS as the internal standard and DMSO- $\mathrm{d}_{6}$ as solvent.

Raman spectroscopy was conducted on a Renishaw InVia Reflex Raman system. The Raman scattering was excited using a diode laser at a wavelength of $785 \mathrm{~nm}$. The laser beam was focused on the sample with a 100 an 0.85 microscope objective. The laser power at the sample was $320 \mathrm{~mW}$. The exposure was $10 \mathrm{~s}$ and two accumulations of Raman measurements were carried out. In addition, an optical microscope was coupled to the system.

The molecular weight and molecular weight distribution of prepared MCC-g-PCL were measured on a Waters 5510 gel permeation chromatograph (U.S.A.). DMSO was used as eluent at a flow rate of $0.5 \mathrm{~mL} \mathrm{~min}{ }^{-1}$ at $50{ }^{\circ} \mathrm{C}$. The SEC system was calibrated with linear polystyrene standards. 


\subsection{Characterization of GO/MCC-g-PCL composite film}

The surface morphology of the nanocomposite films was examined using a scanning electron microscope (S-4800, Hitachi, Japan) operated at an accelerating voltage of $10 \mathrm{kV}$ with a working distance of $20 \mathrm{~mm}$. The films were cut into small pieces and fixed on conductive carbon tape. Moreover, the composite materials were immersed in liquid nitrogen and then fractured in order to analyze their cross-sections by scanning electron microscopy (SEM). The films were then sputtered with a thin layer of gold for $60 \mathrm{~s}$ with a sputter-coating unit.

Fourier transform infrared (FT-IR) spectra were collected with a VERTEX TOV spectrometer (Bruker, Germany), to determine the interfacial bonds in MCC and MCC- $g$-PCL. All spectra were recorded at $2 \mathrm{~cm}^{-1}$ resolution with 32 consecutive scans within the standard wavenumber range from 4000 to $400 \mathrm{~cm}^{-1}$. All the samples were dried under vacuum at $50{ }^{\circ} \mathrm{C}$ for $24 \mathrm{~h}$ before testing.

The thermal stability of MCC, MCC- $g$-PCL and GO/MCC- $g$ PCL films was tested with an STA 449 F3 Jupiter analyzer (NETZSCH, Germany). Samples were heated from $25{ }^{\circ} \mathrm{C}$ to $600{ }^{\circ} \mathrm{C}$ at a constant heating rate of $10{ }^{\circ} \mathrm{C} \mathrm{min}^{-1}$. All the measurements were performed under a nitrogen atmosphere.

Ultraviolet-visible (UV-vis) spectroscopy was applied to study the UV-vis transmittance of MCC, MCC- $g$-PCL and GO/MCC- $g$ PCL films. For each sample, wavelength scanning was performed in three random places along the film. The wavelength was monitored from 200 to $800 \mathrm{~nm}$, and spectra were taken on an Agilent/Varian Cary 5000 UV-vis spectrophotometer.

The tensile strength and elongation-at-break of the films were measured in a conditioned state at $25{ }^{\circ} \mathrm{C}$, using a universal tensile testing machine (ZB-WL300, Hangzhou Zhibang
Automated Instrument Co., Ltd, PR China) with crosshead speed of $5 \mathrm{~mm} \mathrm{~min}^{-1}$. The specimens, measuring $10 \mathrm{~mm} \times 60$ $\mathrm{mm}$, were prepared and tested under ambient conditions. To minimize the experimental error, the tensile tests were carried out at $10 \mathrm{~mm} \mathrm{~min}^{-1}$ on ten individual films for each sample.

\section{Results and discussion}

\subsection{Structure and molecular weight of MCC-g-PCL}

The typical ${ }^{13} \mathrm{C}$ NMR spectrum of MCC- $g$-PCL copolymer is shown in Fig. 2A. The methylene carbon signals of PCL can be observed at $\delta=32.6 \mathrm{ppm}, 33.9 \mathrm{ppm}, 25.5 \mathrm{ppm}, 24.5 \mathrm{ppm}$, $28.1 \mathrm{ppm}, 61.2 \mathrm{ppm}$ and $64.1 \mathrm{ppm}$, ascribed respectively to the carbons in the a, $\mathrm{a}^{\prime}, \mathrm{b}, \mathrm{c}, \mathrm{d}, \mathrm{e}^{\prime}$ and e ring-opened caprolactone positions. MCC-g-PCL displayed typical signals for cellulose. ${ }^{26,27}$ At the same time, the strong signals in the region between 50 and $120 \mathrm{ppm}$ were assigned mainly to the different carbons of cellulose (Fig. 2A). The carbon signals of the cellulose backbone appearing at $\delta=100.9 \mathrm{ppm}$ and $64.1 \mathrm{ppm}$ were assigned to $\mathrm{C} 1$ and $\mathrm{C6}$, respectively, in the anhydrous glucose units (AGU). ${ }^{28}$ Carbons C2, C3 and C5 show a cluster of resonances at 71.5-76.9 ppm in the cross-polarization magic angle spinning (CP/MAS) ${ }^{13} \mathrm{C}$ NMR spectrum. ${ }^{29}$ Moreover, the signal corresponding to the overlap of $\mathrm{C} 6$ bearing a substituted $\mathrm{OH}$ group with the methylene carbon of PCL in the e position appears at $\delta=61.2 \mathrm{ppm}$. The carbon signal at $\delta=99.5 \mathrm{ppm}$ belongs to the $\mathrm{C} 1$ adjacent to the $\mathrm{C} 2$ with substituted $\mathrm{OH}$ groups, indicating PCL grafts at multiple sites of AGU. Especially, the peak at $173.4 \mathrm{ppm}$, identified as the carbonyl carbon signal of the PCL segment (in the f position), confirms successful synthesis of MCC- $g$-PCL. ${ }^{30}$
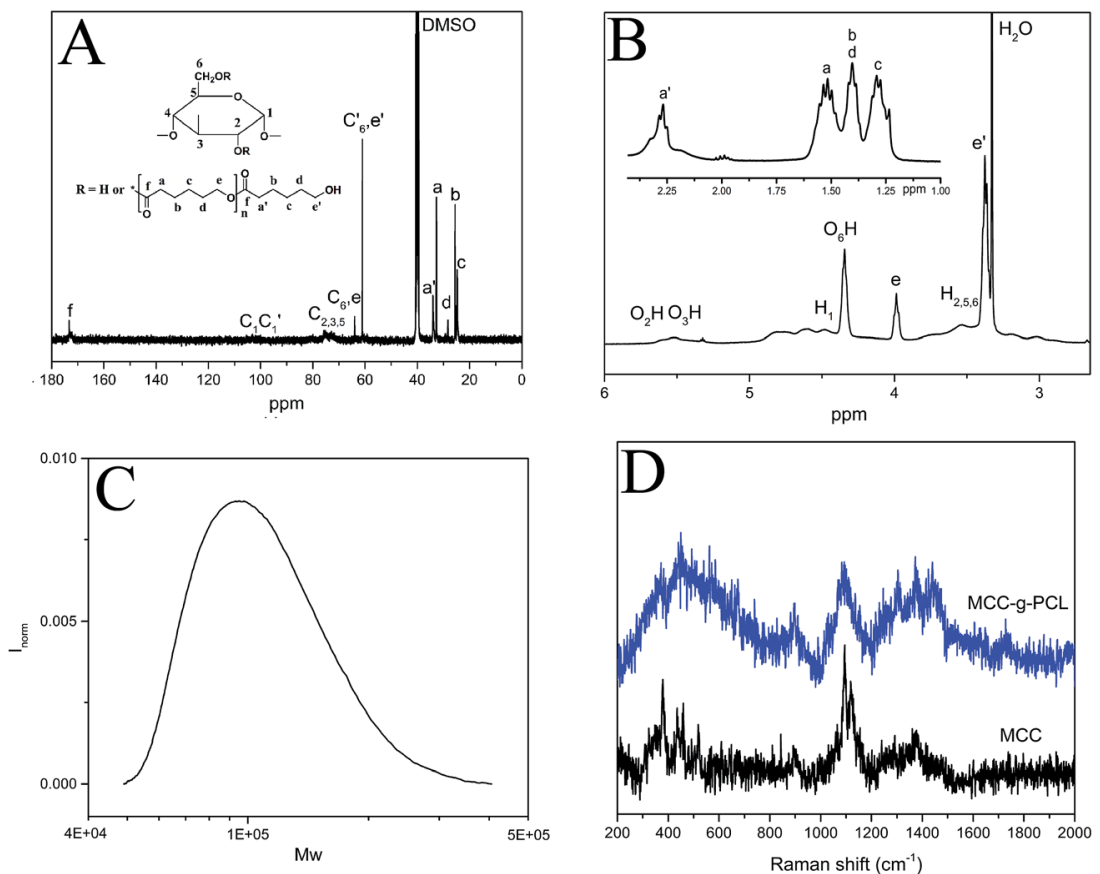

Fig. 2 (A) ${ }^{13} \mathrm{C}$ NMR spectrum, (B) ${ }^{1} \mathrm{H}$ NMR spectrum, (C) gel permeation chromatography trace of MCC-g-PCL, and (D) Raman spectra of MCC and MCC-g-PCL. 
In the ${ }^{1} \mathrm{H}$ NMR spectrum of cellulose- $g$-PCL copolymer (Fig. 2B), the methylene proton peaks of PCL can be observed at $\delta=3.98 \mathrm{ppm}\left(\mathrm{CH}_{2} \mathrm{O}\right.$, e, repeating units), $3.38 \mathrm{ppm}\left(\mathrm{CH}_{2} \mathrm{OH}, \mathrm{e}^{\prime}\right.$, end unit), $2.27 \mathrm{ppm}\left(\mathrm{COCH}_{2}, \mathrm{a}^{\prime}\right.$, end unit), $1.52 \mathrm{ppm}\left(\mathrm{COCH}_{2}\right.$, a, repeating unit), $1.41 \mathrm{ppm}\left(\mathrm{CH}_{2}, \mathrm{~b}, \mathrm{~d}\right)$, and $1.29 \mathrm{ppm}\left(\mathrm{CH}_{2}, \mathrm{c}\right) .{ }^{31}$ The resonance peaks derived from the protons of AGU of the cellulose backbone appear at $\delta=4.47 \mathrm{ppm}$ and $3.53 \mathrm{ppm}$, attributed to H1 and $\mathrm{H} 5,6,2$, respectively. Meanwhile, the signals at $\delta=5.51 \mathrm{ppm}$, $5.37 \mathrm{ppm}$, and $4.35 \mathrm{ppm}$ correspond to the protons of the residual hydroxyl groups of AGU. According to these assignments, the molecular factors of the graft copolymers, including DP, MS, DS and weight content of the PCL side-chain, where MS is the molar substitution of PCL, DS is the degree of substitution of PCL (e.g. the number of PCL side-chains per cellulose unit), DPPCL is the degree of polymerization of PCL, and WPCL is the weight content of PCL side-chains. According to these assignments, the molecular factors of the graft copolymers including the degree of substitution, degree of polymerization, the molar substitution, and the weight content of PCL side chains can be estimated according to previous study. ${ }^{21}$ The appearance of proton signals of caproyl groups in this spectrum, further confirmed that MCC- $g$ PCL copolymers were synthesized successfully.

Fourier transform (FT)-Raman spectra in the frequency region of $2000-200 \mathrm{~cm}^{-1}$ for MCC and MCC- $g$-PCL are shown in Fig. 2D. For both MCC and MCC- $g$-PCL, the characteristic Raman band at $1500-1200 \mathrm{~cm}^{-1}$ was assigned to $\mathrm{C}-\mathrm{H}$ bending. ${ }^{30}$ Additionally, a strong peak located at $1095.5 \mathrm{~cm}^{-1}$ corresponded to the cellulose $\mathrm{C}-\mathrm{O}$ ring stretching ${ }^{32,33}$ with an alternative assignment for the glycosidic linkage (C-O-C) mode. ${ }^{34,35}$ The peak located around $894.9 \mathrm{~cm}^{-1}$ was assigned to mixed modes $(\mathrm{C}-\mathrm{C}-\mathrm{C}, \mathrm{C}-\mathrm{H}-\mathrm{O})$ including angle bending. ${ }^{32}$ The presence of a Raman band at $1740 \mathrm{~cm}^{-1}$, corresponding to carbonyl groups, indicated that the ring-opened caprolactone was successfully introduced into cellulose-OH groups by esterification catalyzed by DMAP. These results confirm the success of the grafting procedure.

The molecular weight of MCC-g-PCL was measured using gel permeation chromatography (Fig. 2C). The determined molecular weight of MCC- $g$-PCL is significantly higher than the molecular weight of native cellulose, which may be due to functionalization. The data verify that the presented cellulose grafting is mild and does not result in pronounced degradation of the cellulose backbone.

\subsection{Morphology of composite materials}

To study the influence of GO on the microstructure of MCC- $g$ PCL, the nanocomposite films were investigated by field emission scanning electron microscopy (FE-SEM). It can be observed that the pure MCC- $g$-PCL film with some long voids exhibits a clean but rough surface (Fig. 3(A3)), showing weak interaction between the MCC-g-PCL molecules, owing to the decreased numbers of hydroxyl groups in the cellulose chains and the hindrance of the PCL side-chains. In comparison with the pure MCC-g-PCL film, a regular uniform network structure (Fig. 3(B1)) of GO/MCC- $g$-PCL nanohybrids is observed clearly, which is expected to enhance their mechanical properties. The results show that the uniform network benefited from the excellent dispersion of GO nanohybrids within the MCC- $g$-PCL matrix, which is much smoother than the pure MCC- $g$-PCL film (Fig. 3(B3)). Therefore, the GO oxygenated groups might be interacting with the PCL chains, providing good compatibility between the GO filler and the polymer.

\subsection{Contact angle}

Since the ultimate goal of this study is to disperse as finely as possible the prepared GO composite materials in a hydrophobic polyester matrix, contact angle measurements were used to estimate the change in hydrophobicity of the grafted substrates compared with unmodified MCC, and a distinct difference in hydrophobicity was observed (Fig. 4). As shown in Fig. 4, the contact angle increases from $39.6^{\circ}$ for the pure MCC film to $87.3^{\circ}$ for the MCC- $g$-PCL, namely the MCC- $g$-PCL displays a higher hydrophobic nature, ${ }^{36}$ because of the shielding of PCL chains grafted onto the OH-rich hydrophilic surface of MCC. ${ }^{37}$ It should be noted that addition of GO did not cause a reduction in hydrophobicity of the MCC- $g$-PCL-based film's surface; on the contrary, the contact angle of GO/MCC-g-PCL film increased a little. Hence, GO/MCC- $g$-PCL composite materials showed

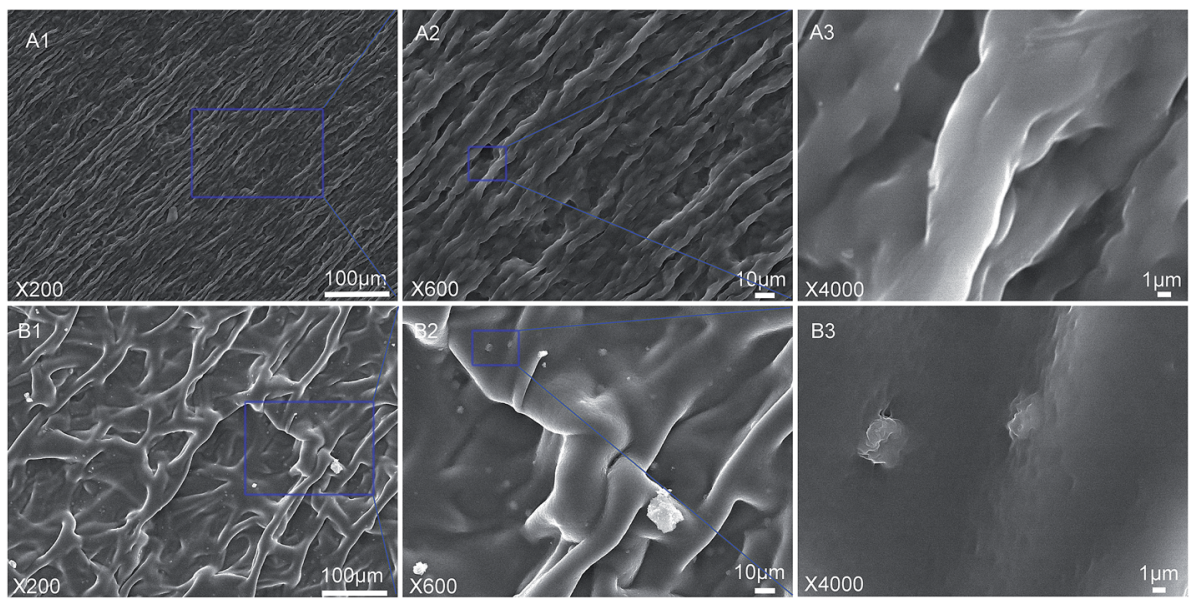

Fig. 3 Scanning electron micrographs of (A) MCC-g-PCL and (B) GO/MCC-g-PCL films. 


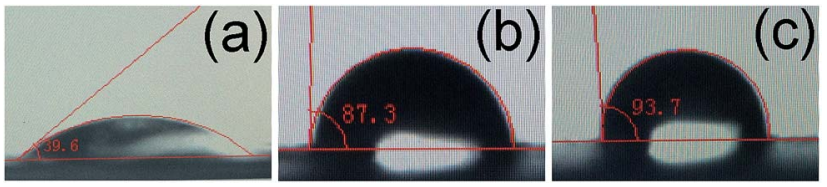

Fig. 4 Contact angles of (a) MCC, (b) MCC-g-PCL and (c) GO/MCC$g$-PCL films.

a more hydrophobic nature as the hydroxyls of the MCC-g-PCL interacted with that of GO. This can be understood in light of the apparent irregular roughness of the GO/MCC-g-PCL film compared with MCC- $g$-PCL, distinguished from the surface SEM images of GO/MCC-g-PCL film and MCC- $g$-PCL film (Fig. 4), which increased the contact angle. Another possible reason is that the hydroxyl groups in GO are mostly occupied owing to its interaction with MCC- $g$-PCL. ${ }^{38}$ And the results are in accordance with that of $\mathrm{rGO} / \mathrm{CNC}$ hybrid fibers as well. ${ }^{39}$ Moreover, the addition of GO sheets also influenced the porosity and pore size distribution of the membrane, which had an effect on the hydrophobicity of the film as well.

\subsection{FT-IR analysis}

The FT-IR spectra of MCC, MCC- $g$-PCL and GO/MCC- $g$-PCL films are displayed in Fig. 5. Compared with the unmodified precursor, MCC film, MCC- $g$-PCL film showed a strong band at $1729 \mathrm{~cm}^{-1}$ which was assigned to the typical carbonyl group stretching. Other obvious transmittance bands located at about $2935 \mathrm{~cm}^{-1}$ and $2861 \mathrm{~cm}^{-1}$ correspond to C-H stretching of $\mathrm{CH}_{3}$ and $\mathrm{CH}_{2}$ groups, respectively. And the peak located at $1156 \mathrm{~cm}^{-1}$ is associated with $\mathrm{C}-\mathrm{H}$ bending. ${ }^{40,41}$ FT-IR measurement also revealed the interactions between MCC- $g$ PCL matrix and GO filler. It seemed that the chemical surface composition of the composite films was not obviously affected by the introduction of GO but there were some differences.

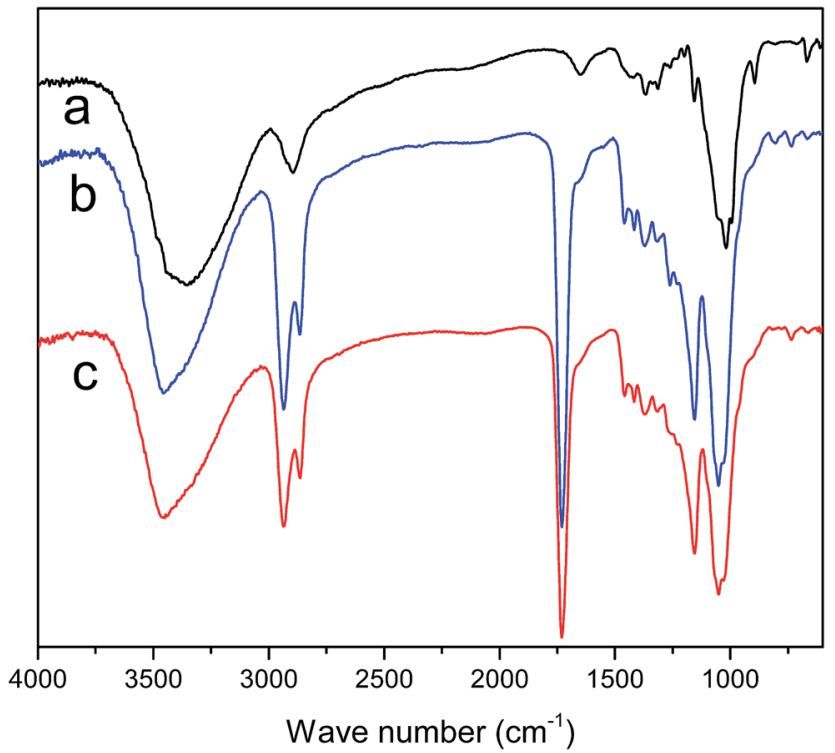

Fig. 5 FT-IR spectra of (a) MCC, (b) MCC-g-PCL and (c) GO/MCC-g$P C L$ films.
Compared with MCC-g-PCL film, the broadened peak at $3447 \mathrm{~cm}^{-1}$ and the relative intensity changes of the $\mathrm{C}-\mathrm{O}$ stretching vibrations in the GO/MCC-g-PCL composites imply the disturbance of hydrogen-bonding in MCC- $g$-PCL, indicating the formation of intermolecular hydrogen bonds and excellent compatibility between GO and MCC- $g$-PCL. ${ }^{42-45}$

\subsection{Thermal stability}

The thermogravimetric analysis (TGA) curves (Fig. 6) showed a one-step profile of weight loss for neat MCC, MCC-g-PCL and GO/MCC-g-PCL composites, which was related to the degradation of carbonyl groups and the chains of cellulose. ${ }^{46}$ It was observed that the polymer grafting improved the thermal stability of the film. Thermal degradation of neat MCC, which was due to depolymerization, dehydration and decomposition of cellulosic glycosyl units, ${ }^{47}$ started at $256^{\circ} \mathrm{C}$ and reached its maximum value $\left(T_{\max }\right)$ at $329{ }^{\circ} \mathrm{C}$. Polymer-grafted MCC started to decompose at a temperature of approximately $205^{\circ} \mathrm{C}$, which was substantially lower than that of the pristine MCC sample. The MCC-g-PCL film displayed a slower degradation rate between 250 and $400{ }^{\circ} \mathrm{C}$ and a higher residual mass at high temperature compared with MCC. The enhanced thermal stability of MCC-g-PCL may originate from the entanglement of the PCL side-chains, which provided indirect confirmation of successful grafting of polymer brushes on MCC. It is clearly seen that the TGA curves of pure MCC- $g$-PCL and GO/ MCC- $g$-PCL films are practically overlapping, indicating that the thermal decomposition behavior of GO/MCC- $g$-PCL is almost identical to that of neat MCC- $g$-PCL films, suggesting a strong interaction between GO sheets and MCC-g-PCL chains. This may be due to the effect of nanoclay on the polymer matrix, and was similar to the effect of GO on thermal properties of $\mathrm{GO} /$ cellulose and $\mathrm{GO} /$ chitosan films. $^{48,49}$

\subsection{Mechanical properties}

The mechanical properties of MCC, MCC- $g$-PCL and the GO/MCC- $g$-PCL composite are compared in Table 1. Neat MCC

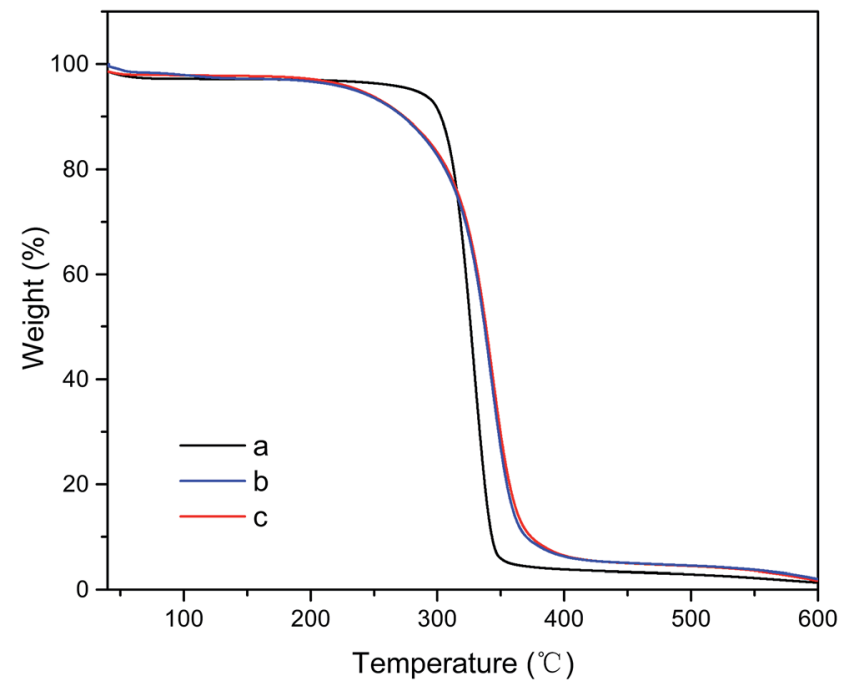

Fig. 6 Thermogravimetric analysis plots for (a) MCC, (b) MCC-g-PCL and (c) GO/MCC-g-PCL films. 
Table 1 Mechanical properties of composite films

\begin{tabular}{llcl}
\hline Sample & $\begin{array}{l}\text { Tensile } \\
\text { strength }(\mathrm{MPa})\end{array}$ & $\begin{array}{l}\text { Elongation- } \\
\text { at-break }(\%)\end{array}$ & $\begin{array}{l}\text { Contact } \\
\text { angle }\left(^{\circ}\right.\end{array}$ \\
\hline MCC & $9.53 \pm 0.44$ & $3.6 \pm 0.33$ & 39.6 \\
MCC- $g$-PCL & $2.63 \pm 0.18$ & $6.4 \pm 0.29$ & 93.7 \\
GO/MCC- $g$-PCL & $4.55 \pm 0.15$ & $15.5 \pm 0.51$ & 87.3
\end{tabular}

film was stiff with a tensile strength of $9.63 \mathrm{MPa}$ and a strain at breaking of $3.6 \%$. As observed from the results of mechanical analysis, MCC- $g$-PCL exhibited poor tensile strength of $2.63 \mathrm{MPa}$ and a higher elongation-at-break of $6.4 \%$ as compared with the pristine MCC film, which may be due to the inner plasticization effect of PCL side-chains. The GO/MCC- $g$-PCL film showed distinct improvement of the tensile strength compared with MCC- $g$-PCL film. The film with $1.0 \mathrm{wt} \%$ GO had a tensile strength of $4.55 \mathrm{MPa}$, which was enhanced by $73.0 \%$ compared with that of pure MCC- $g$-PCL (2.63 MPa), attributed to the increased intermolecular hydrogen-bonding between the hydroxyl groups of MCC- $g$-PCL and hydroxyl or carboxyl groups of GO. In addition, the MCC- $g$-PCL acted as glue and GO sheets acted as reinforcement, the two playing a coordinated role. This may be due to the improved interfacial strength between PCL side-chains and GO. The GO sheets present a bigger surface area which could provide greater geometric constraints for the randomness of polymer molecules, ${ }^{50}$ providing improved surface interlocking with the PCL matrix, which has already been confirmed from SEM micrographs. The elongation-atbreak of GO/MCC-g-PCL was increased from $6.4 \%$ up to $\mathbf{1 5 . 5 \%}$, which was similar to the values for epoxy composites filled with both graphene oxide (GO) and diglycidyl ether of bisphenol-A functionalized GO (DGEBA-f-GO) sheets and regenerated cellulose/graphite oxide. ${ }^{51,52}$ This increase in elongation of the composites was related with the origin of GO platelet structure. On the other hand, it may be attributed to the excellent dispersion of GO, enhancing the interfacial adhesion provided by the grafted PCL chains. ${ }^{53,54}$ More precisely, it has been previously suggested that grafted chains create a cocontinuous phase in which local stresses are evenly transferred to the remaining PCL phase, which in turn improves the mechanical properties of the bulk material. ${ }^{55}$ Further, the hydroxyl end-group on PCL grafted onto the MCC reacted with the carboxyl group of GO sheets, which significantly increased the adhesion at the interface. These results, combined with the fact that cellulose-based composite film containing GO provided better mechanical properties than neat MCC- $g$-PCL film, agree with those obtained by Han et al., who prepared cellulose/graphite oxide composite film to study its mechanical behavior. ${ }^{51}$

\subsection{UV-vis transmittance}

The light transmittance of MCC, MCC- $g$-PCL and GO/MCC- $g$ PCL composite films was measured using UV-vis spectroscopy, and the results are shown in Fig. 7. Pure MCC film exhibited light transmittance higher than $91 \%$ across the visible light

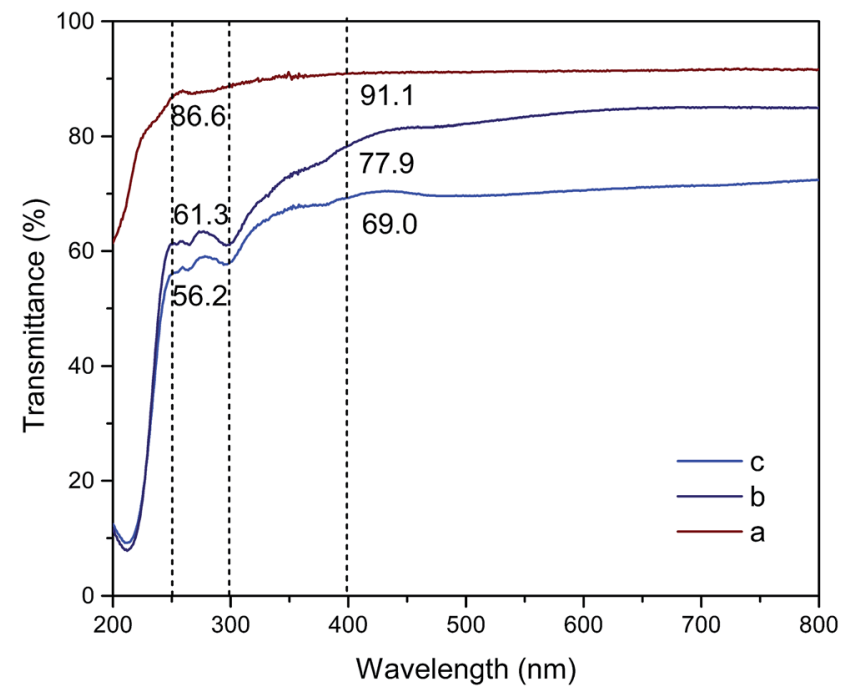

Fig. 7 UV-vis spectra of (a) MCC, (b) MCC-g-PCL and (c) GO/MCC-gPCL hybrid films.

range (400-800 $\mathrm{nm}$ ), and it did not demonstrate an anti-UV property as it presented a transmittance of over $60 \%$ even at $200 \mathrm{~nm}$. In contrast to the findings for CA film reported in a previous study, ${ }^{15}$ our results showed that cellulose grafted with polycaprolactone film without incorporation of GO had good ability to shield against UV light. Notably, the MCC- $g$-PCL film possessed good UV-shielding capacity at 200-400 nm compared with MCC films. Therefore, a novel material with enhanced light-shielding capacity and good optical transparency in the visible light range was obtained through the modification of MCC into MCC- $g$-PCL. GO/MCC- $g$-PCL composite films offered an improved high-energy lightshielding capacity as compared with MCC-g-PCL film, especially at 250-400 $\mathrm{nm}$. Particularly for UVC irradiation $(250 \mathrm{~nm})$, the GO/MCC- $g$-PCL film containing $1.0 \mathrm{wt} \%$ GO displayed a UV-shielding capacity of $43.8 \%$, combined with $70.2 \%$ optical transparency under visible light. These results are in accordance with a previous study which reported that cellulose films showed reduced optical transmittance (from $91.5 \%$ to $78.2 \%$ ) after blending with $0.4 \mathrm{wt} \%$ grapheme, ${ }^{56}$ revealing an effective anti-UV property with comparable transparency, which was competitive with transparent cellulose nanocrystals (CNC)/polylactic acid (PLA) and GO/cellulose acetate (CA) films, ${ }^{15,57}$ especially at wavelengths between 300 and $400 \mathrm{~nm}$. In addition, although surface-functionalized zirconia nanoparticles/poly(butanediol monoacrylate) films had better UV-shielding capacity than the GO/MCC-g-PCL film prepared in the present study, their transmittance of visible light was very low. ${ }^{58}$

\section{Conclusions}

In the present study, we demonstrated an efficient method for the facile preparation of MCC- $g$-PCL in a TBAA/DMSO solvent system in conjunction with $\varepsilon$-caprolactone, and characterized the structure and molecular weight. Furthermore, we also 
demonstrated an easy method of preparing a biocomposite from MCC-g-PCL and GO that served as a good example of ultraviolet-shielding, and was more environmental friendly. The GO introduced into the composite remarkably influenced both the inherent structure of the MCC- $g$-PCL and the surface morphology of the film. Moreover, the GO/MCC-g-PCL composite showed significantly improved mechanical properties compared with those of the pure MCC- $g$-PCL film, owing to the strong hydrogen-bonding interaction that physically crosslinked GO with MCC- $g$-PCL, while no changes of thermal stability were found. Moreover, GO/MCC-g-PCL composite films containing $1.0 \mathrm{wt} \%$ GO offered an effective high-energy lightshielding capacity for light between $200 \mathrm{~nm}$ and $300 \mathrm{~nm}$ in wavelength. This technique may lead to new and convenient methods for the production of various modified forms of cellulose and the processing of new composite materials from previously incompatible components.

\section{Conflicts of interest}

There are no conflicts to declare.

\section{Acknowledgements}

The authors are grateful for the support of the Project of Scientific Research Special Fund for Public Industry in Forestry (201504602-2).

\section{Notes and references}

1 A. Gao, C. Zhang, K. Song and A. Hou, Carbohydr. Polym., 2014, 114, 392-398.

2 M. Gorjanc, K. Jazbec, M. Šala, R. Zaplotnik, A. Vesel and M. Mozetič, Cellulose, 2014, 21(4), 3007-3021.

3 K. Jazbec, M. Šala, M. Mozetič, A. Vesel and M. Gorjanc, J. Nanomater., 2015, 16(1), 1-9.

4 D. Zhang, L. Chen, D. Fang, G. William Toh, X. Yue, Y. Chen and H. Lin, Text. Res. J., 2013, 83(15), 1625-1633.

5 J. D'Orazio, S. Jarrett, A. Amaro-Ortiz and T. Scott, Int. J. Mol. Sci., 2013, 14(6), 12222-12248.

6 N. Deeba, I. Mohammad, S. Maria and M. Hasan, Curr. Drug Targets, 2013, 14(10), 1128-1134.

7 M. Montazer and A. Maali, J. Phys. Chem. B, 2014, 118(6), 1453-1470.

8 W. Sricharussin, P. Threepopnatkul and N. Neamjan, Fibers Polym., 2011, 12(8), 1037-1041.

9 L. Xu, G. Zheng, J. Miao and F. Xian, Appl. Surf. Sci., 2012, 258(19), 7760-7765.

10 M. Montazer, M. Amiri and M. Malek, Photochem. Photobiol., 2013, 89(5), 1057-1063.

11 A. Becheri, M. Dürr, P. Nostro and P. Baglioni, J. Nanopart. Res., 2008, 10(4), 679-689.

12 Ş. Uğur, M. Sarışık, H. Aktaş, Ç. Uçar and E. Erden, Nanoscale Res. Lett., 2010, 5(7), 1204.

13 A. Yadav, V. Prasad, A. Kathe, S. Raj, D. Yadav, C. Sundaramoorthy and N. Vigneshwaran, Bull. Mater. Sci., 2006, 29(6), 641-645.
14 R. Yang, P. Christensen, T. Egerton and J. White, Polym. Degrad. Stab., 2010, 95(9), 1533-1541.

15 A. C. de Moraes, P. F. Andrade, A. F. de Faria, M. B. Simoes, F. C. Salomao, E. B. Barros, C. Goncalves Mdo and O. L. Alves, Carbohydr. Polym., 2015, 123, 217-227.

16 Y. Teramoto, M. Yoshioka, N. Shiraishi and Y. Nishio, J. Appl. Polym. Sci., 2002, 84(14), 2621-2628.

17 Y. Teramoto and Y. Nishio, Polymer, 2003, 44(9), 2701-2709.

18 R. Shi and H. Burt, J. Appl. Polym. Sci., 2002, 89, 718-727.

19 N. Lin, G. Chen, J. Huang, A. Dufrense and P. R. Chang, J. Appl. Polym. Sci., 2009, 113(5), 3417-3425.

20 A. Mujica-Garcia, S. Hooshmand, M. Skrifvars, J. M. Kenny,

K. Oksman and L. Peponi, RSC Adv., 2016, 6, 9221-9231.

21 Y. Guo, X. Wang, Z. Shen, X. Shu and R. Sun, Carbohydr. Polym., 2013, 92, 77-83.

22 Y. Habibi, A. Goffin, N. Schiltz, E. Duquesne, P. Dubois and A. Dufresne, J. Mater. Chem., 2008, 18, 5002.

23 K. Beyaz, M. Charton, A. Rouilly, E. Vedrenne, C. VacaGarcia, A. Benaboura and S. Thiebaud-Roux, Ind. Crops Prod., 2017, 97, 32-40.

24 L. Xu, X. Lu and X. Cheng, RSC Adv., 2015, 5, 7902279030.

25 I. Ikeda, K. Washino and Y. Maeda, Sen'i Gakkaishi, 2003, 59(3), 110-114.

26 M. Ibrahim, W. El-Zawawy and M. Nassar, Carbohydr. Polym., 2010, 79, 694-699.

27 N. Lin, J. Huang, P. Chang, J. Feng and J. Yu, Carbohydr. Polym., 2011, 83, 1834-1842.

28 C. Yan, J. Zhang, Y. Lv, J. Yu, J. Wu, J. Zhang and J. He, Biomacromolecules, 2009, 10(8), 2013-2018.

29 H. Kono, S. Yunoki, T. Shikano, M. Fujiwara, T. Erata and M. Takai, J. Am. Chem. Soc., 2002, 124, 7506-7511.

30 A. Mayumi, T. Kitaoka and H. Wariishi, J. Appl. Polym. Sci., 2006, 102(5), 4358-4364.

31 C. Jiang, X. Wang, P. Sun and C. Yang, Int. J. Biol. Macromol., 2011, 48(1), 210-214.

32 R. Atalla and S. Nagel, Science, 1974, 185, 522-523.

33 K. Kong and S. J. Eichhorn, J. Macromol. Sci., Part B: Phys., 2005, 44, 1123-1136.

34 N. Wanasekara, A. Michud, C. Zhu, S. Rahatekar, H. Sixta and S. Eichhorn, Polymer, 2016, 99, 222-230.

35 H. Edwards, D. Farwell and D. Webster, Spectrochim. Acta, Part A, 1997, 53, 2383-2392.

36 H. Youssef, G. Anne-Lise, S. Nancy, D. Emmanuel, D. Philippe and D. Alain, J. Mater. Chem., 2008, 18(41), 5002-5010.

37 G. Chen, A. Dufresne, J. Huang and P. Chang, Macromol. Mater. Eng., 2009, 294(1), 59-67.

38 R. Faradilla, G. Lee, J. Roberts, P. Martens, M. Stenzel and J. Arcot, Cellulose, 2018, 25(1), 399-416.

39 G. Chen, T. Chen, K. Hou, W. Ma, M. Tebyetekerwa, Y. Cheng, W. Weng and M. Zhu, Carbon, 2018, 127, 218227.

40 H. Dong, Q. Xu, Y. Li, S. Mo, S. Cai and L. Liu, Colloids Surf., $B, 2008,66(1), 26-33$.

41 Q. Xu, J. Kennedy and L. Liu, Carbohydr. Polym., 2008, 72(1), 113-121. 
42 S. Gan, S. Zakaria and S. Jaafar, Carbohydr. Polym., 2017, 172, 284-293.

43 T. Kondo, J. Polym. Sci., Part B: Polym. Phys., 1994, 32(7), 1229-1236.

44 Y. Feng, X. Zhang, Y. Shen, K. Yoshinoc and W. Feng, Carbohydr. Polym., 2012, 87(1), 644-649.

45 L. Yao, Y. Lu, Y. Wang and L. Hu, Carbon, 2014, 69, 552-562.

46 A. de Oliveira, M. Ferrarezi, I. Yoshida and M. Goncalves, J. Appl. Polym. Sci., 2012, 123(4), 2027-2035.

47 M. Roman and W. Winter, Biomacromolecules, 2004, 5, 16711677.

48 X. Zhang, X. Liu, W. Zheng and J. Zhu, Carbohydr. Polym., 2012, 88(1), 26-30.

49 D. Han, L. Yan, W. Chen and W. Li, Carbohydr. Polym., 2011, 83(2), 653-658.

50 Y. Pan, T. Wu, H. Bao and L. Li, Carbohydr. Polym., 2011, 83(4), 1908-1915.
51 D. Han, L. Yan, W. Chen, W. Li and P. Bangal, Carbohydr. Polym., 2011, 83(2), 966-972.

52 Y. Wan, L. Tang, L. Gong, D. Yan, Y. Li, L. Wu, J. Jiang and G. Lai, Carbon, 2014, 69, 467-480.

53 H. Lönnberg, K. Larsson, T. Lindström, A. Hult and E. Malmström, ACS Appl. Mater. Interfaces, 2011, 3(5), 1426-1433.

54 N. Lin, G. Chen, J. Huang, A. Dufrense and P. R. Chang, J. Appl. Polym. Sci., 2009, 113(5), 3417-3425.

55 X. Cao, Y. Habibi and L. Lucia, J. Mater. Chem., 2009, 19, 7137-7145.

56 X. Zhang, X. Liu, W. Zheng and J. Zhu, Carbohydr. Polym., 2012, 88(1), 26-30.

57 J. Gwon, H. Cho, S. Chun, S. Lee, Q. Wu and S. Lee, RSC Adv., 2016, 6, 9438-9445.

58 S. Scholz and S. Kaskel, J. Colloid Interface Sci., 2008, 323(1), 84-91. 